One can judge from the present paper the little value of the Weierstrassian formulas when the applications of the general theory are involved or whenever any kind of numerical computation is desired. One sees also how easy it is to introduce errors in the calculation. The book of Lévy already mentioned is in most respects an excellent work, certainly from the standpoint of applied mathematics, much of the material being new; but when a substitution involving an elliptic function in the Weierstrassian form is introduced, the book is not free from errors. For example, not to mention many inaccuracies, besides the mistake already cited, it is seen that on page 82 of Lévy's book $e_{1}+e_{2}+e_{3} \neq 0$. The same error is found on page 156, while in the calculation of $\zeta u$, the functions introduced on page 104 are incorrectly given. At the end of my larger work, volume 1, the Weierstrassian functions are put in juxtaposition with those of the older theory and it is seen that thereby nothing new is added.

UnIVERSITY OF CINCINNATI, June 14, 1918.

\title{
ON PLANE ALGEBRAIC CURVES WITH A GIVEN SYSTEM OF FOCI.
}

BY PROFESSOR ARNOLD EMCH.

(Read before the American Mathematical Society, April 27, 1918.)

1. Let

$$
\phi(u, v, w)=0
$$

be a curve of class $n$, and

$$
u \xi+v \eta+w \zeta=0
$$

a line with the coordinates $u, v, w$, and $x^{\prime}=\xi / \zeta, y^{\prime}=\eta / \zeta$ current cartesian coordinates. Then

$$
-1 \cdot \xi-i \cdot \eta+(x+i y) \xi=0
$$

is a line which passes through the point $(x, y)$ and the circular point $I$ with the slope $i$. The coordinates of (3) are $u=-1$, 
$v=-i, w=x+i y$. Consequently, if these satisfy (1), then the line (3) will be a tangent of (1); and as it passes through the point $(x, y)$, this point will be a focus of the curve. Hence, to find the real foci of (1), we have to determine real values of $x$ and $y$ in such a manner that

$$
\phi(-1,-i, x+i y)=0 \text {. }
$$

In other words, we have to solve the equation of the $n$th degree in $w$,

$$
\phi(-1,-i, w)=0 .
$$

The $n$ complex roots of this equation interpreted in the cartesian plane are the required foci.* These are also obtained by writing (4) in the form $f(x, y)+i g(x, y)=0$, where $f$ and $g$ are real polynomials in $x$ and $y$, and by finding the intersections of $f$ and $g$. In this manner we get $n^{2}$ solutions of which $n$ are real and correspond to the $n$ roots of (5). The remaining $n(n-1)$ imaginary solutions are the associates of the $n$ roots of (5).

2. Conversely, when the foci $z_{1}, z_{2}, \cdots, z_{n}$ of a curve are given in a complex plane, we can easily form the equation

$$
\prod_{k=1}^{n}\left(z-z_{k}\right)=z^{n}+a_{1} z^{n-1}+a_{2} z^{n-2}+\ldots+a_{n}=0,
$$

with these as roots. The curve of the $n$th class with these as foci has the form

$$
\begin{aligned}
\phi \equiv & w^{n}+\left(\alpha_{11} v+\alpha_{12} u\right) w^{n-1}+\left(\alpha_{21} v^{2}+\alpha_{22} v u+\alpha_{23} u^{2}\right) w^{n-2} \\
& +\left(\alpha_{31} v^{3}+\alpha_{32} v^{2} u+\alpha_{33} v u^{2}+\alpha_{34} u^{3}\right) w^{n-3}+\cdots \\
& +\alpha_{n 1} v^{n}+\alpha_{n 2} v^{n-1} u+\alpha_{n 3} v^{n-2} u^{2}+\ldots+\alpha_{n n} u^{n}=0 .
\end{aligned}
$$

The foci are conversely obtained by putting in this equation $u=-1, v=-i$, and solving for $w$. In this manner from (7) we get

$$
\begin{aligned}
w^{n}+ & \left(i \alpha_{11}-\alpha_{12}\right) w^{n-1}+\left(-\alpha_{21}+i \alpha_{22}+\alpha_{23}\right) w^{n-2} \\
& +\left(i \alpha_{31}+\alpha_{32}-i \alpha_{33}-\alpha_{34}\right) w^{n-3}+\cdots \\
& +(-i)^{n} \alpha_{n 1}-(-i)^{n-1} \alpha_{n 2}+(-i)^{n-2} \alpha_{n 3}+\cdots \\
& +(-1)^{n} \alpha_{n n}=0 .
\end{aligned}
$$

* Siebeck: "Ueber eine neue analytische Behandlungsweise der Brennpunkte," Crelle's Journal, vol. 64, pp. 175-182 (1865). 
In this equation we can determine real values of the $\alpha_{j k}$ $(j, k=1,2,3, \cdots, n)$ in an infinite number of ways in such a manner that (8) becomes identical with (6). For any such a set of $\alpha_{j k}$ 's (7) represents a curve of the $n$th class with the given points as foci.

To obtain the coordinates of (7) in projective point coordinates $\xi, \eta, \zeta$, we must eliminate $w, v, u$ between (7) and the three equations

$$
\xi=\frac{\partial \phi}{\partial u}, \quad \eta=\frac{\partial \phi}{\partial v}, \quad \zeta=\frac{\partial \phi}{\partial w},
$$

as is well known.

3. When an $n$-ic $F(x, y)=0$ is given, the foci may be found by making the substitution $y=i x+c$, and by establishing the discriminant for the equation $F(x, i x+c)=0$ in $x$. This leads to an equation in $c$ whose roots determine the foci. If $c_{k}=A_{k}+i B_{k}$ is such a root, then, as $c=y-i x$, the coordinates of the corresponding focus are $x=-B_{k}, y=A_{k}$.

Another method for finding the foci of an $n$-ic is suggested by the foregoing results: Establish the equation $\phi(u, v, w)=0$ corresponding to $F(x, y)=0$ by the well known method of elimination, as suggested above for the converse case.

4. As an example consider the circular cubic*

$$
F \equiv\left(x^{2}+y^{2}\right) x+y z^{2}=0,
$$

whose cartesian equation is obtained by putting $z=1$. The equation of the equivalent curve of class (6), in line coordinates $u, v, w$ is obtained by eliminating $x, y, z$ between (10) and

$$
u=\partial F / \partial x=3 x^{2}+y^{2}, \quad v=2 x y+z^{2}, \quad w=2 y z .
$$

After this rather tedious process the required equation becomes

$$
\begin{aligned}
4 w^{6}+24 u v w^{4}-\left(u^{4}-30 u^{2} v^{2}-27 v^{4}\right) & w^{2} \\
& -4 u^{3} v\left(u^{2}+v^{2}\right)=0 .
\end{aligned}
$$

Setting $u=-1, v=-i$, this reduces to

$$
w^{6}+6 i w^{4}-w^{2}=0,
$$

\footnotetext{
* See Annals of Mathematics, vol. 14, pp. 57-71 (1912).
} 
whose roots are $w_{1}, 2 ; 3,4= \pm \frac{1}{2} \sqrt{2}(1+i)(-3 \pm \sqrt{8})$, $w_{5}=0, w_{6}=0$. The coordinates of the foci $F_{i}$ of the circular cubic (10) are therefore

$$
\begin{array}{lll}
\boldsymbol{F}_{1}\left(x_{1}=y_{1}=2-\frac{3}{2} \sqrt{2}\right), & F_{2}\left(x_{2}=y_{2}=-2+\frac{3}{2} \sqrt{2}\right), \\
F_{3}\left(x_{3}=y_{3}=-2-\frac{3}{2} \sqrt{2}\right), & F_{4}\left(x_{4}=y_{4}=2+\frac{3}{2} \sqrt{2}\right), \\
F_{5}\left(x_{5}=y_{5}=0\right), & F_{6}\left(x_{6}=y_{6}=0\right) .
\end{array}
$$

$F_{5}$ and $F_{6}$ coincide with the origin, and form a quadruple focus which lies on the curve. It is called quadruple, since it may be considered as the intersection of two two-fold tangents at the isotropic points.

The foci $F_{1}, F_{2}, F_{3}, F_{4}$ of a circular cubic lie on a circle, which in this case degenerates into a straight line, the bisecting line of the first and third quadrant.

5. Confocal conics.-Let $F_{1}(1,0)$ and $F_{2}(-1,0)$ be the foci of a system of confocal conics. Then the determining equation is $w^{2}-1=0$, and the system of curves of the second class with these foci is

$$
w^{2}-\alpha u^{2}-(\alpha-1) v^{2}=0 .
$$

The corresponding cartesian equation is

$$
\frac{x^{2}}{\alpha}+\frac{y^{2}}{\alpha-1}=1 \text {, }
$$

which, for a variable $\alpha$, represents a system of confocal conics.

6. A certain class of curves, admitting the nth roots of unity as foci. The equation determining these foci is

$$
w^{n}-1=0,
$$

and the corresponding curve of class $n$ has the form

$$
\begin{aligned}
w^{n}-\left(\alpha v^{n}+\alpha_{1} v^{n-1} u+\right. & \alpha_{2} v^{n-2} u^{2} \\
& \left.+\alpha_{3} v^{n-3} u^{3}+\cdots+\alpha_{n} u^{n}\right)=0
\end{aligned}
$$

in which, when $n$ is even, say $n=2 \lambda$,

$$
\alpha(-1)^{\lambda}+\alpha_{2}(-1)^{\lambda-1}+\alpha_{4}(-1)^{\lambda-2}+\cdots=1 .
$$


For an even $\lambda$, this becomes

$$
\alpha-\alpha_{2}+\alpha_{4}-\cdots=1 \text {. }
$$

For any $\lambda$, there is $\alpha_{1}-\alpha_{3}+\alpha_{5}-\cdots=0$. When $\lambda$ is odd, then $-\alpha+\alpha_{2}-\alpha_{4}+\cdots=1$. When $n$ is odd, say $n=2 \lambda$ +1 , then $\alpha-\alpha_{2}+\alpha_{4}-\cdots=0$, and $\alpha_{1}-\alpha_{3}+\alpha_{5}-\cdots$ $=1$, when $\lambda$ is odd; $-\alpha_{1}+\alpha_{3}-\alpha_{5}+\cdots=1$, when $\lambda$ is even.

We shall, in particular, consider the case where (15) has the form

$$
w^{n}-v^{2 k} \cdot u^{n-2 k}=0,
$$

in which $n$ and $k$ must both be either even or odd in order that (16) may reduce to (14) for $u=-1, v=-\ddot{i}$.

After a rather complicated process of elimination the cartesian equation of this special class of curves with the $n$th roots of unity as foci becomes

$$
x^{n-2 k} \cdot y^{2 k}=(-1)^{n-2 k} \cdot \frac{(2 k)^{2 k}}{n^{n} \cdot(n-2 k)^{2 k-n}},
$$

which is an $n$-ic. For $n=3, k=1$, we get the cubic hyperbola $x y^{2}=-4 / 27$. The condition for a proper $n$-ic in (17) is evidently $n-2 k \geqq 1, n \geqq 3$.

UNIVERSITY OF ILLINOIS.

\title{
QUADRATIC SYSTEMS OF CIRCLES IN NON-EUCLIDEAN GEOMETRY.
}

\author{
BY PROFESSOR D. M. Y. SOMMERVILLE.
}

(Read before the American Mathematical Society, October 26, 1918.)

$\S 1$. The equation of any circle can be written

$$
k S-\alpha^{2}=0,
$$

where $S=0$ is the equation of the absolute, and

$$
\alpha \equiv l x+m y+n z=0
$$

is the equation of the axis, the center being the absolute polar of this line. 